\title{
Polarization properties of polymer-based photonic crystal fibers
}

\author{
D. Poudereux ${ }^{*}$, K. Mileńko ${ }^{2}$, A. Dybko ${ }^{3}$, J. M. Otón ${ }^{1}$, and T. R Woliński ${ }^{2}$ \\ ${ }^{1}$ CEMDATIC, ETSI Telecomunicación, Universidad Politécnica de Madrid,Av. Complutense 30, 28040 Madrid, \\ Spain \\ ${ }^{2}$ Faculty of Physics, Warsaw University of Technology, Koszykowa 75, 00-662 Warszawa, Poland \\ ${ }^{3}$ Faculty of Chemistry, Warsaw University of Technology, Noakowskiego 3, 00-664 Warszawa, Poland
}

Received June 12, 2014; accepted June 30, 2014; published June 30, 2014

\begin{abstract}
Selectively filled photonic crystal fibers with polydimethylsiloxane (PDMS), a silicon-type material, have been studied. It has been demonstrated that polarization properties of these hybrid devices change and so do the properties of guided light in relation with temperature, finding that the state of polarization (SOP) changes with an increasing temperature but remains constant for a wide spectrum of wavelengths for a determinate temperature.
\end{abstract}

Photonic crystal fibers (PCFs) are versatile optical fibers that can be designed for specific purposes. The design of these fibers has no limits and could be manufactured in any shape depending on the desired purpose. Moreover, PCFs can be filled with a liquid or gaseous materials improving their inherent optical properties or acquiring new ones as in the case of photonic liquid crystal fibers (PLCFs). The infiltration of PCF air holes with different materials yields novel hybrid all-fiber optical devices. Various applications can be developed with these new tunable and hybrid devices such as fiber-optic sensors, filters, and switches for optical communications [1-5]. When the material infiltrating the cladding holes of a solid-core PCF has its refractive index higher than that of the fiber core, the light guiding mechanism changes from index guiding to the photonic band-gap (PBG) effect. The PBG guiding mechanism is also presented in a PCF with a hollow core. Then light is guided in an air channel and consequently, the refractive index of the core is lower than that of the cladding. In this case only certain values of wavelengths are being guided and the others are irradiated out through the fiber cladding.

As PCFs have periodically arranged air holes in the cladding, it is possible to fill selectively some of the holes while keeping the others empty. In this way a different configuration could be developed based on the same "guest" fiber and the same infiltrating "host" material, changing only the order and the number of filled cladding holes.

One of the material candidates to be infiltrated inside a PCF is polydimethylsiloxane (PDMS). Although the primary field of application of this silicone elastomer is embedding electronic components generally used in micro- and nanotechnologies, PDMS is often used in fluidics [6-9], optical systems [10], and sensors [11-12]. It has been previously presented [13] that PDMS could be used to develop a high-birefringence PCF without postprocessing [14].

In this work we present polarization properties of two different PCFs selectively infiltrated with PDMS.

Polarization-maintaining PCF (PM-PCF) manufactured by Blaze Photonics in pure silica has two big holes of $4.5 \mu \mathrm{m}$ in the same diagonal close to the solid core of $4.5 \mu \mathrm{m}$ and 5 rings of smaller holes of $2.2 \mu \mathrm{m}$ in the diameter. The outer diameter of the PM-1550-01 PM-PCF is $125 \mu \mathrm{m}$ and it was manufactured to operate as a singlemode fiber in a third communications window at $1500 \mathrm{~nm}$ with highly reduced temperature sensitivity (Fig. 1, left).
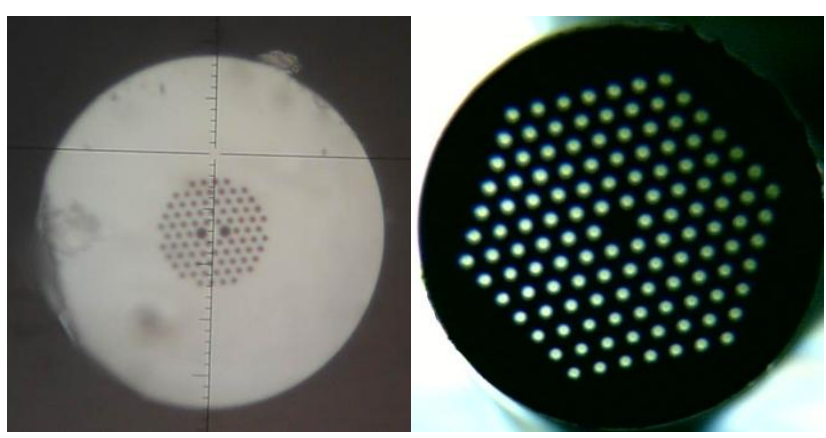

Fig. 1. Photonic crystal fibers: PM-1550-01 (left) and LMA-25 (right).

LMA25 PCF fabricated by NKT Photonics (Fig. 2, right) was made in pure silica and has a solid core of $25 \mu \mathrm{m}$ designed to operate as endlessly single-mode (ESM) with a large mode area. This fiber has an external diameter of $258 \mu \mathrm{m}$.

In the case of the PM-1550-01 only the bigger holes have been selectively filled with PDMS. Before the infiltration the smaller holes in the cladding were collapsed with a standard fusion splicer, therefore only two big holes have been infiltrated (Fig. 2, left).

*E-mail: dpoudere@gmail.com 

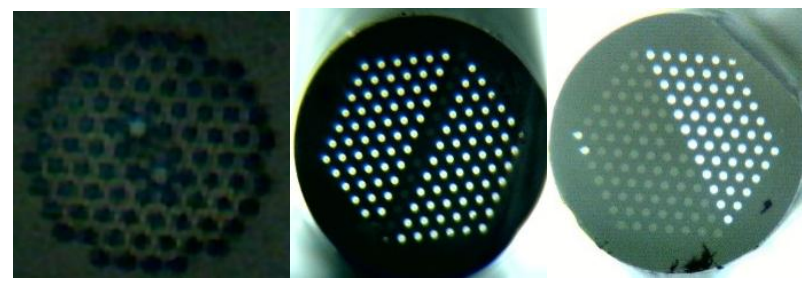

Fig. 2. PM-1550-01 selectively filled with PDMS (left); LMA-25 selectively filled with PDMS (middle and right).

In the case of LMA25 the selected holes were blocked one by one with an optical adhesive and then filled with PDMS. Two different configurations have been developed: one in which only one central row is filled (Fig. 2, middle) and the other in which half of the fiber is filled/empty (Fig. 2, right). Note that in this last configuration the fiber is only symmetric in the axis perpendicular to the interphase of the filled/empty sides.

To study polarization changes with a temperature of the PM-1550-01 fiber, a tunable laser operating in the range of $1500-1600 \mathrm{~nm}$ connected to a polarization controller PAT 9000, has been used. By changing the external temperature it is possible to observe a change of the state of polarization (SOP) in the output light (Fig. 3).
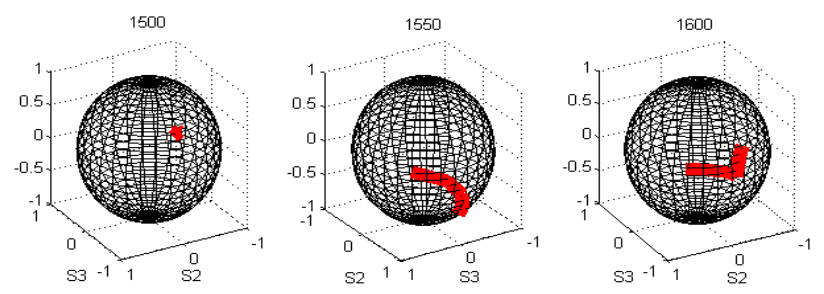

Fig. 3. Changes of SOP at 1500, 1550, and $1600 \mathrm{~nm}$ inthe PM-1550-01 fiber selectively filled with PDMS for 5 different temperatures in a temperature range between $20^{\circ} \mathrm{C}$ and $80^{\circ} \mathrm{C}$

The change in the SOP depends on the wavelength (Fig.3) and it is related with the phase difference between two orthogonal polarizations. If the slope of both effective reflective indices is the same at one determinate wavelength, there will be no measurable changes in the SOP (Fig. 3a). However, if at another wavelength the slope of both indices is different, it will produce a change in the SOP.

The SOP measured at $1550 \mathrm{~nm}$ for a continuous change in temperature in the range of $20^{\circ} \mathrm{C}$ to $110^{\circ} \mathrm{C}$ is presented in Fig. 4.

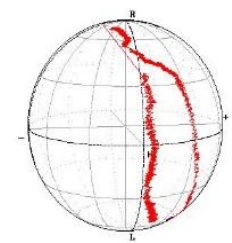

Fig. 4. SOP change with temperature in the PM-1550 PCF at $1550 \mathrm{~nm}$.
The SOP is changing with rising temperature and hysteresis is observed when the sample is cooled. This could be caused by the internal stress of PDMS induced by heating.

In both cases of the LMA25 fiber selectively filled, the SOP changes with temperature at each wavelength, but in some temperatures, the SOP remains constant for all wavelengths (Figs. 5 and 6).
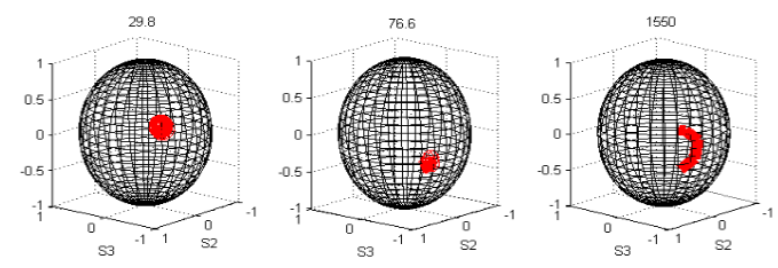

Fig. 5. LMA 25 - one row configuration: left and middle - changes in the SOP at $29.8^{\circ} \mathrm{C}$ and $76.8^{\circ} \mathrm{C}$ in the wavelength range between $1500 \mathrm{~nm}$ and $1640 \mathrm{~nm}$; right- changes in the SOP at $1550 \mathrm{~nm}$ in the temperature range between $20^{\circ} \mathrm{C}$ and $90^{\circ} \mathrm{C}$.
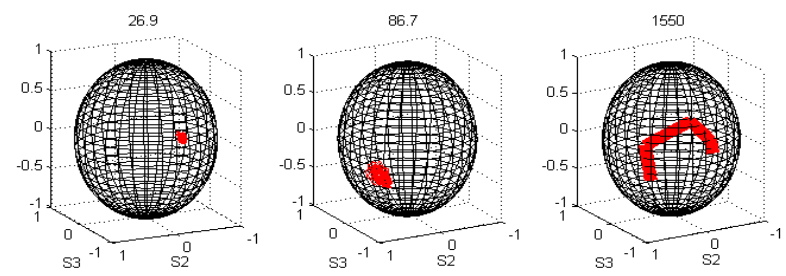

Fig. 6. LMA 25 - half configuration; left and middle -changes in SOP at $26.9^{\circ} \mathrm{C}$ and $86.7^{\circ} \mathrm{C}$ in the wavelength range between $1500 \mathrm{~nm}$ and $1640 \mathrm{~nm}$;right- changes in SOP at $1550 \mathrm{~nm}$ in the temperature range between $20^{\circ} \mathrm{C}$ and $90^{\circ} \mathrm{C}$.

In some temperatures, the SOP in the LMA25 fiber remains constant for all wavelengths between 1500 and $1640 \mathrm{~nm}$. This behavior is even stronger in half the configuration and could be explained as polarization behavior, when in selected temperatures no birefringence exists because only one polarization mode is guided.

The LMA25 fiber with half infiltration was placed between crossed polarizers at $45^{\circ}$ with respect to the azimuth of the fiber to measure birefringence caused by the asymmetrical structure of the infiltration pattern. A white light source was coupled in one end of the fiber and the output was measured with a spectrometer. The interference pattern and its change with increasing temperature was observed (Fig. 7). 


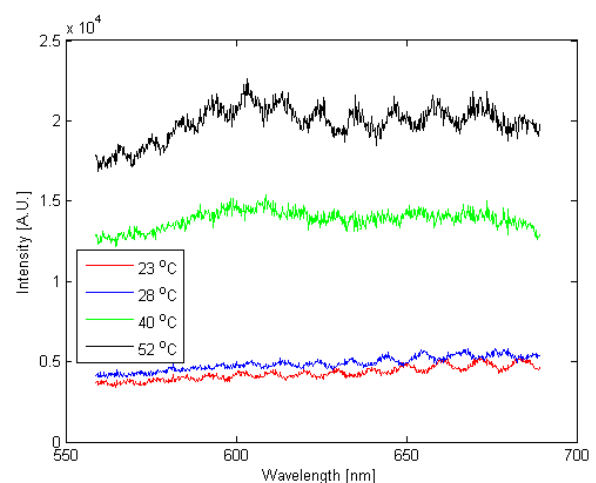

Fig. 7. Interferometricpattern measured in LMA25 fiber half-filled with PDMS at selected temperatures.

The birefringence is defined either by the phase modal birefringence $B(\lambda)$ or by the group birefringence $G(\lambda)$, and can be expressed as:

$$
\begin{gathered}
B=\frac{\lambda\left(\beta_{y}-\beta_{x}\right)}{2 \pi} \\
G=B-\lambda \frac{d B}{d \lambda}=\frac{\bar{\lambda}^{2}}{L \Delta \lambda}
\end{gathered}
$$

If the phase modal birefringence is independent on the wavelength, the derivative term in Eq. (2) vanishes and both group and phase modal birefringences are equal [15]. In this case, by measuring the space between the neighboring peaks it is possible to measure $\mathrm{B}(\lambda)$. For $23^{\circ} \mathrm{C}$ the measured birefringence is $9.47 \times 10^{-4}$ and $8.25 \times 10^{-4}$ at $60^{\circ} \mathrm{C}$. For the temperatures in the range of $35^{\circ} \mathrm{C}-45^{\circ} \mathrm{C}$ the contrast of the interferometry effect is minimal. This effect will be investigated deeply in the near future with a mathematical model.

In conclusion, two different fibers have been filled selectively with PDMS and their polarization properties along with thermal tunability have been studied. It was shown that PDMS is a good candidate for manufacturing hybrid devices together with PCFs for polarization controlling or sensing.

To improve the study of the LMA25 fiber selectively filled with PDMS, it is desirable to conduct theoretical modeling in the near future.

This work was supported by the Polish National Science Centre (NCN) under the grant no. 2011/01/B/ST7/05015.

\section{References}

[1] T. Larsen, A. Bjarklev, D. Hermann, J. Broeng, Opt. Expr. 11(20), 2589 (2003).

[2] H.Y. Choi, M.J. Kim, B.H. Lee, Opt. Expr. 15(9), 5711 (2007).

[3] B. Dong, J. Hao, Z. Xu, Opt. Fiber Technol. 17(3), 233 (2011).

[4] D. Poudereux, P. Corredera, E. Otón, J. M. Otón, X.Q. Arregui, Opt. Pura Apl. 46(4), 321 (2013).

[5] P. Lesiak, G. Rajan,Y. Semenova,G. Farrell, A. Boczkowska, A. Domanski, T. Wolinski, Phot. Lett. Poland 2(3), 140 (2010).

[6] M.A. Unger, H.-P. Chou, T. Thorsen, A. Scherer, S.R. Quake, Science 288(5463), 113 (2000).

[7] T. Thorsen, S.J. Maerkl, S.R. Quake, Science 298(5593), 580 (2002).

[8] K. Hosokawa, R. Maeda, J. Micromechanics Microengineering 10(3), 415 (2000).

[9] D.C. Duffy, O.J.A. Schueller, S.T. Brittain, G.M. Whitesides, J. Micromechanics Microengineering 9(3), 211 (1999).

[10] J. Chen, W. Wang, J. Fang, K. Varahramyan, J. Micromechanics Microengineering 14(5), 675 (2004).

[11] H. Lee, S.-I. Chang, E. Yoon, J. Microelectromechanical Syst. 15(6), 1681 (2006).

[12] H. Kudo, T. Sawada, E. Kazawa, H. Yoshida, Y. Iwasaki, K. Mitsubayashi, Biosens. Bioelectron. 22(4), 558 (2006).

[13] C. Markos, K. Vlachos, G. Kakarantzas, Proc. SPIE 7914, 791427 (2011).

[14] C. Kerbage, B. Eggleton, Opt. Expr. 10(5), 246 (2002).

[15] A. Barlow, D.N. Payne, IEEE J. Quantum Electron. 19(5), 834 (1983). 